\title{
Parkes Weber Syndrome and Spinal Arteriovenous Malformations
}

W e read with great interest the article written by Alomari et $\mathrm{al},{ }^{1}$ and agree with the authors that Klippel-Trenaunay syndrome is not associated with spinal cord AVM. However, in their discussion, in relationship to spinal cord AVM with Parkes Weber syndrome, they comment briefly that "Spinal AVM has not been reported to be a feature of Parkes Weber syndrome."

Here, we demonstrate a case of Parkes Weber syndrome harboring a concomitant spinal AVM and raise the argument that spinal AVM may be a feature of Parkes Weber syndrome. A baby girl was born with multiple skin capillary malformations on her right buttock, thigh, shin, and left axilla, as well as overgrowth of the affected right leg. A picture of the lower extremities at age 2 years and 11 months shows the capillary malformations and the overgrowth of the right leg (Fig 1). Results on sonography examination of her right buttock were suggestive of an arteriovenous shunt, which was confirmed by angiography (Fig 2). An angiogram of the right common iliac artery demonstrated microarteriovenous fistulas with an early draining vein in the gluteal area (Fig 2, arrow). Screening MR imaging of the spine at birth demonstrated spinal AVM, which was later confirmed by angiography (Fig 3).

We support the association of spinal AVM with Parkes Weber syndrome for the following reasons:

1) Parkes Weber syndrome and spinal AVM are 2 rare conditions; therefore, it is extremely unlikely that these conditions may coexist by chance.

2) Many older studies erroneously interpret Parkes Weber syndrome as a variant of Klippel-Trenaunay syndrome, under the name "Klippel-Trenaunay-Weber syndrome." For example, the http://dx.doi.org/10.3174/ajnr.A3717 "prototype" article by Djindjian et al, cited by Alomari et al, described 5 cases of Klippel-Trenaunay-Weber syndrome. ${ }^{2}$ Of these 5 cases, 4 actually harbored an arteriovenous fistula in the affected limb, highly suggestive of the diagnosis of Parkes Weber syndrome. Many other reports can be found with the same misunderstanding.

3) Both Parkes Weber syndrome and spinal AVM are associated with a $R A S A 1$ gene mutation. ${ }^{3,4}$

Therefore, although we do not have solid evidence, we hypothesize and propose an association between Parkes Weber syndrome and spinal AVM.

\section{REFERENCES}

1. Alomari AI, Orbach DB, Mulliken JB, et al. Klippel-Trenaunay syndrome and spinal arteriovenous malformations: an erroneous association. AJNR Am J Neuroradiol 2010;31:1608-12

2. Djindjian M, Djindjian R, Hurth M, et al. Spinal cord arteriovenous malformations and the Klippel-Trenaunay-Weber syndrome. Surg Neurol 1977;8:229-37

3. Revencu N, Boon LM, Mulliken JB, et al. Parkes Weber syndrome, vein of Galen aneurysmal malformation, and other fast-flow vascular anomalies are caused by RASA 1 mutations. Hum Mutat 2008;29:959-65

4. Thiex R, Mulliken JB, Revencu N, et al. A novel association between RASA1 mutations and spinal arteriovenous anomalies. AJNR Am J Neuroradiol 2010;31:775-79 Center for Endovascular Treatment, Division of Neuroendovascular Surgery Jichi Medical University Tochigi-ken, Japan S. Nemoto Department of Endovascular Surgery Tokyo Medical and Dental University Tokyo, Japan 


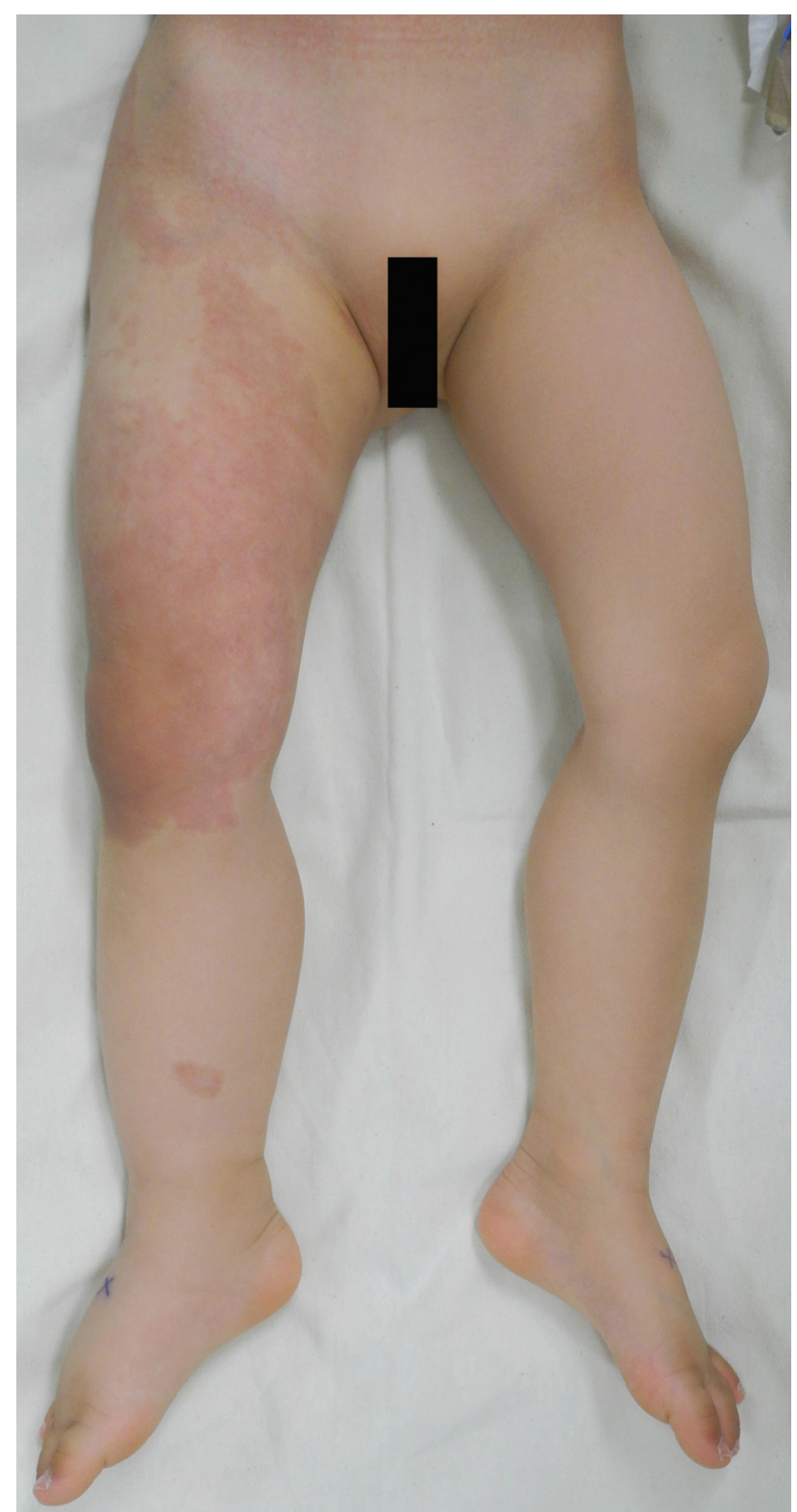

FIG 1. A picture of the lower extremities in a young girl at age 2 years and 11 months shows patchy and multiple capillary malformations on the right thigh and shin. She also had the same capillary malformations on the right buttock and left axilla (not shown). Note the overgrowth of the affected right leg.

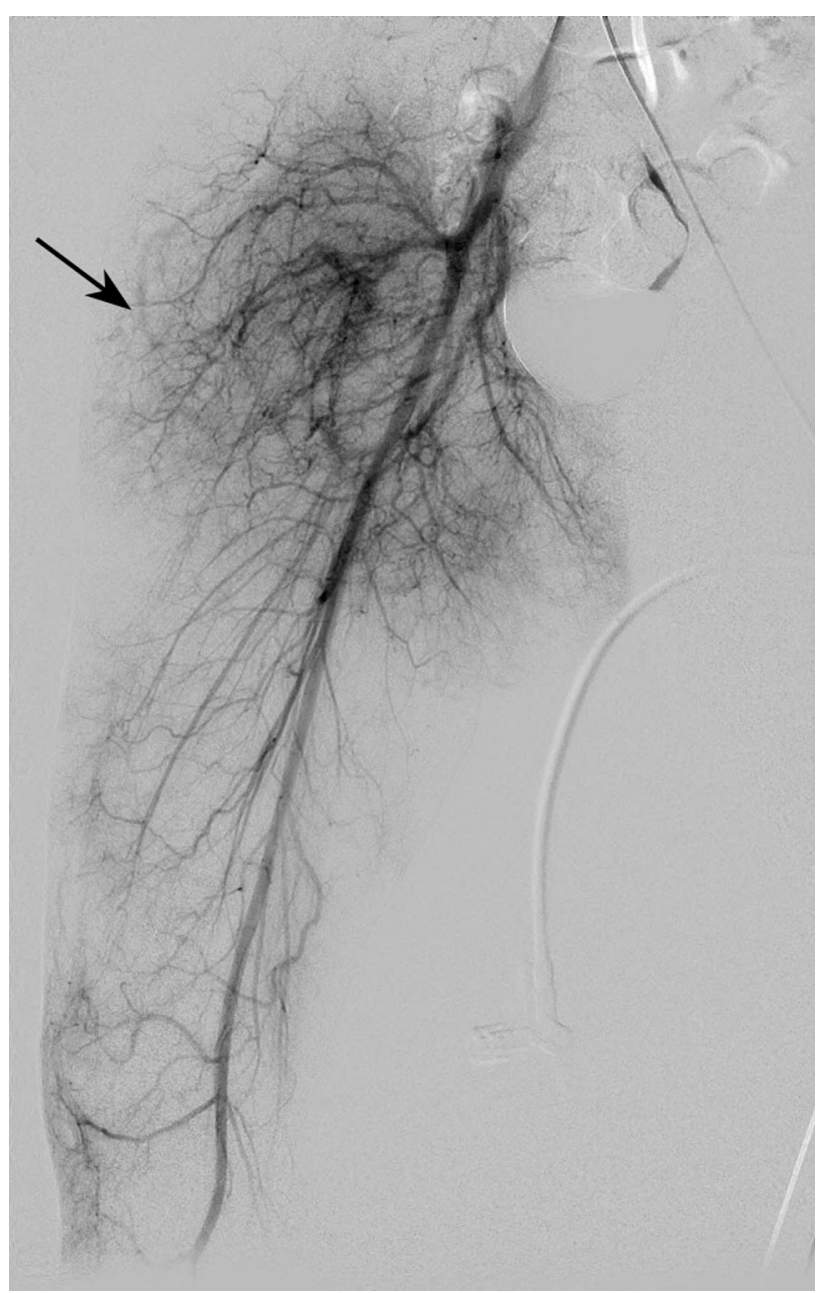

FIG 2. Angiogram of the right common iliac artery in the anteroposterior view in our young patient at age 2 years and 11 months demonstrates multiple microarteriovenous fistulas in the buttock area supplied by the superior and inferior gluteal arteries. Note the early draining vein (arrow). 


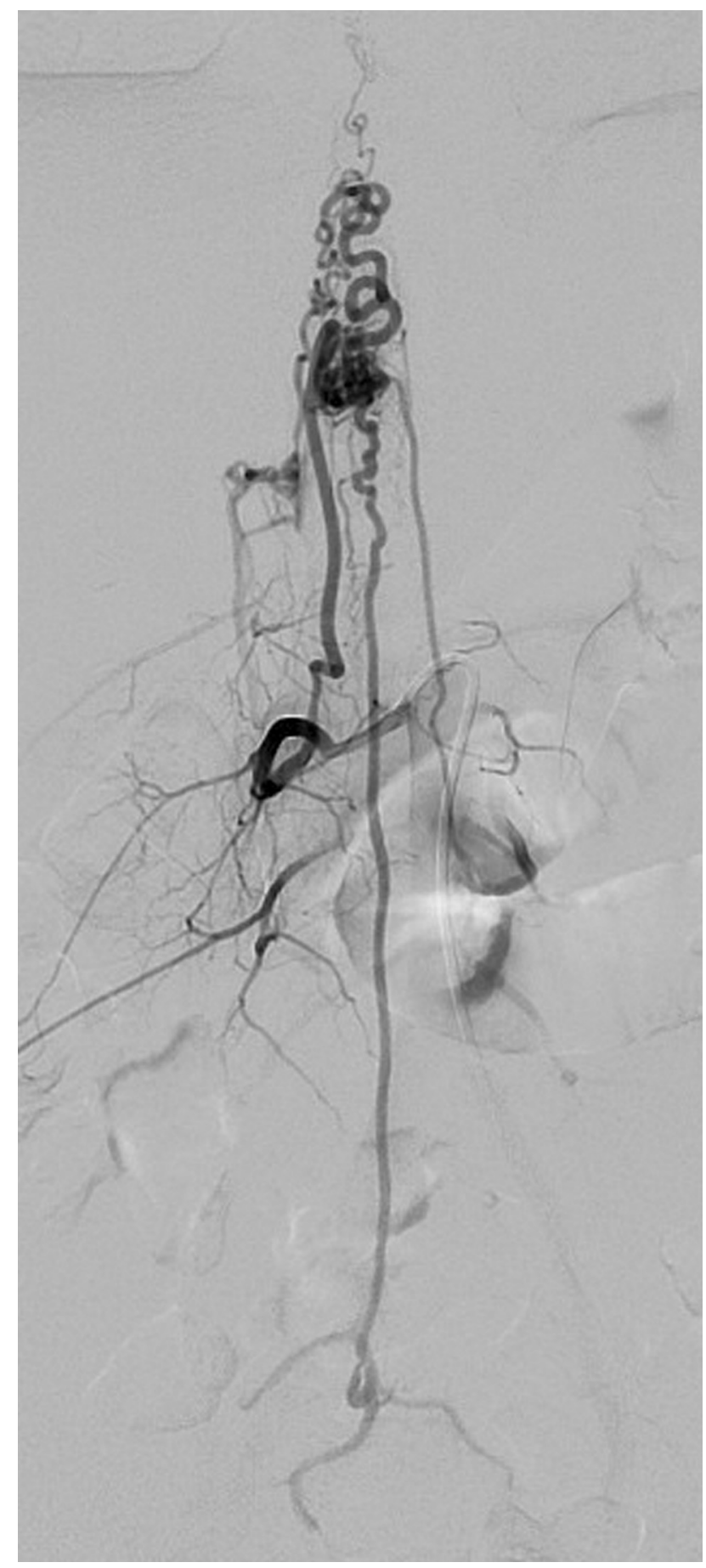

FIG 3. Spinal angiogram performed in our patient at age 2 years and 11 months. Right L2 lumbar artery injection in the anteroposterior view shows spinal cord AVM supplied from the radiculospinal artery. Not shown are the indirect supplies from the right L3 and left L2 radiculospinal arteries. 